\title{
A model to predict $S-N$ curves for surface and subsurface crack initiations in different environmental media
}

\author{
Guian Qian, Chengen Zhou, Youshi Hong* \\ State Key Laboratory of Nonlinear Mechanics, Institute of Mechanics, Chinese Academy of Sciences, Beijing 100190, China
}

\section{A R T I C L E I N F O}

\section{Article history:}

Received 11 August 2013

Received in revised form 5 November 2013

Accepted 8 November 2013

Available online 22 November 2013

\section{Keywords:}

Very-high-cycle fatigue

Aqueous environment

Fatigue life

Fatigue crack initiation

Structural steel

\begin{abstract}
A B S T R A C T
The influence of environmental media on crack propagation of a structural steel at high-cycle and veryhigh-cycle fatigue (VHCF) regimes was investigated with the fatigue tests in air, water and $3.5 \% \mathrm{NaCl}$ aqueous solution. The fatigue strength in water and $3.5 \% \mathrm{NaCl}$ solution is significantly decreased and the cracking morphology due to different driving forces is presented. A model is proposed to explain the influence of environmental media on fatigue life, which reflects the variation of fatigue life with applied stress, grain size, inclusion size and material yield stress. The model prediction is in good agreement with experimental observations.
\end{abstract}

(c) 2013 Elsevier Ltd. All rights reserved.

\section{Introduction}

Very-high-cycle fatigue (VHCF) [1], (also named ultra-high-cycle fatigue [2], ultra-long-life fatigue [3], or gigacycle fatigue [4]) of metallic materials is generally regarded as fatigue failure at stress levels below conventional fatigue limit and the corresponding fatigue life beyond $10^{7}$ loading cycles. Lots of modern engineering structures and components, such as airplanes, turbines, nuclear structures, automobiles and high speed trains are expected to have fatigue life in the range of $10^{7}$ to $10^{10}$ load cycles.

One typical feature of VHCF for high strength steels is that the $S-N$ curve consists of two parts corresponding to subsurface and surface crack initiation, resulting in a stepwise or duplex shape [5-11]. Generally, the crack initiation in VHCF regime is observed as fisheye pattern on fracture surface, which is located at specimen subsurface region and originated from a nonmetallic inclusion for high strength steels [3,5-16]. Since the pioneering work performed by Naito et al. [17,18] and by Atrens et al. [19], there have been a variety of studies on the VHCF behavior for different materials. Among these studies, the crack initiation mechanism in VHCF attracted most of the attention. Murakami et al. [3] attributed the mechanism of subsurface crack initiation to the interaction of hydrogen embrittlement with cyclic damage. Bathias and Paris [4] found that subsurface crack initiation originated from either nonmetallic inclusions or other microstructural inhomogeneities, e.g. perlite colonies and long platelets. They [4] argued that the

\footnotetext{
* Corresponding author. Tel.: +86 1082543966 .

E-mail address: hongys@imech.ac.cn (Y. Hong).
}

probability of finding a sufficient stress concentration inhomogeneity is much higher in the interior of the material than at the surface. Nishijima and Kanazawa [15] attributed the reason why the fatigue life for internal failure is longer than that for surface failure to the fact that the stress intensity factor for flaws with the same size in the material interior is smaller than that at the surface. The influence of some factors, such as loading frequency $[12,14,20,21]$, surface finishing condition [8,16,22], material microstructure state [23-25] and environmental media [10,11,26], on the VHCF properties of high strength steels has been widely studied. Among them, the effect of ultrasonic frequency on the estimated fatigue strength has been intensively studied in order to make sure that the fatigue results obtained by using ultrasonic testing and conventional fatigue equipment with a low frequency are comparable. Stanzl-Tschegg and Mayer [20] showed that the frequency influences might be divided into intrinsic and extrinsic. The former one is related to strain rate, dislocation structures, crack formation and propagation. Whereas, the latter influence includes the correlation of test frequency with environmental effect, influence of creep, specimen heating during ultrasonic testing, etc. However, Furuya et al. [14] found that the loading frequency does not have significant impact on the VHCF behavior of a high strength steel. Recently, Zhao et al. [12] showed that loading frequencies do have effect on fatigue strength of materials, but for materials with some specific microstructure the resultant of the effect may defer. Loading frequencies have little influence on specimens with high strength, while for the specimens with low tensile strength the fatigue resistance is markedly high in ultrasonic testing. 


\section{Nomenclature}

\begin{tabular}{|c|c|}
\hline$a$ & crack depth \\
\hline CIS & critical inclusion size \\
\hline$E$ & Young's modulus \\
\hline$F$ & geometrical factor for stress intensity factor calculation \\
\hline HV & Vickers hardness \\
\hline$k$ & $\begin{array}{l}\text { resistance of dislocation movement, corresponding to } \\
\text { material yield stress }\end{array}$ \\
\hline$k_{\mathrm{w}}$ & $w_{\mathrm{i}} / w_{\mathrm{s}}$ \\
\hline$K_{\mathrm{I}}$ & stress intensity factor \\
\hline$K_{\mathrm{IC}}$ & fracture toughness of material \\
\hline$l$ & grain radius \\
\hline$N_{\mathrm{f}}$ & fatigue failure cycles \\
\hline$N_{\mathrm{i}}$ & fatigue cycles required for crack initiation at subsurface \\
\hline$N_{\mathrm{s}}$ & fatigue cycles required for crack initiation at surface \\
\hline$n_{\mathrm{i}}$ & $\begin{array}{l}\text { normalized fatigue cycles required for crack initiation at } \\
\text { subsurface }\end{array}$ \\
\hline$n_{\mathrm{s}}$ & $\begin{array}{l}\text { normalized fatigue cycles required for crack initiation at } \\
\text { surface }\end{array}$ \\
\hline$r$ & inclusion radius \\
\hline$r_{\mathrm{p}}$ & plastic zone \\
\hline
\end{tabular}

$\begin{array}{ll}\Delta \widetilde{U} & \text { dimensionless unit increment of energy } \\ \Delta U_{\mathrm{i}} & \text { unit increment of energy for subsurface crack initiation } \\ \Delta U_{\mathrm{s}} & \text { unit increment of energy for surface crack initiation } \\ w_{\mathrm{i}} & \text { surface energy related to subsurface crack initiation } \\ w_{\mathrm{s}} & \text { surface energy related to surface crack initiation } \\ \alpha & \begin{array}{l}0.813 \text { for surface, } 0.528 \text { for subsurface and } 0.969 \text { for } \\ \text { interior inclusions }\end{array} \\ \sigma_{\max } & \text { maximum applied stress } \\ \sigma_{\mathrm{s}} & \text { yield stress } \\ \sigma_{\mathrm{a}} & \text { applied stress } \\ \sigma_{\max }^{\mathrm{w}} & \text { applied maximum stress for the tests in fresh water } \\ \sigma_{\max }^{\mathrm{s}} & \text { applied maximum stress for the tests in } 3.5 \% \text { NaCl aque- } \\ & \text { ous solution } \\ \sigma_{\max }^{\mathrm{a}} & \text { applied maximum stress for the tests in laboratory air } \\ \Delta \sigma & \text { stress amplitude } \\ \varphi & 0.5 \Delta \sigma / \mathrm{k} \\ \psi & \quad r / l \\ v & \text { Poisson's ratio } \\ \mu & \text { shear modulus }\end{array}$

Shiozawa and Lu [16] found that for surface shot-peened specimens, due to the surface residual stress induced by shot peening subsurface crack initiation dominated. We [8] studied the influence of surface notch on VHCF behavior of a structural steel, which revealed that surface notch decreases the fatigue strength and the possibility of subsurface crack initiation. Krupp et al. [25] studied the effect of the microstructure of an austenitic-ferritic duplex steel on the VHCF behavior and revealed that the formation of slip bands caused by fatigue damage in VHCF regime leads to the initiation and propagation of microstructurally short cracks in a very localized manner. Lei et al. [5] found that the inclusion size and location has a significant impact on VHCF behavior for high strength steels. The degradation of VHCF strength caused by the increase of inclusion size is ascribed to the decrease of the critical stress of fine-granular-area (FGA) formation for large inclusions. We [10] investigated the effect of environmental media on the fatigue strength and crack initiation of a high strength steel in VHCF regime and the decrease of fatigue strength in environmental media is reported. However, the crack initiation and propagation process of high strength steels in environmental media in VHCF regime is still not clear.

In addition to experimental investigations, theoretical or empirical models for fatigue strength and life prediction in VHCF regime are of significant importance for both scientific and engineering applications. Murakami et al. [3] developed a model to predict fatigue strength in VHCF regime based on crack initiation site, crack area and material hardness. Hong et al. [6] demonstrated that the formation of FGA is responsible for a majority part of total fatigue life. It is shown in [27-29] that in VHCF regime the crack growth constitutes insignificant portion of the total fatigue life. Instead, the importance of fatigue crack initiation stage has been repeatedly emphasized.

Chapetti et al. [30] showed a relation between the FGA size, the inclusion size and the fatigue life by fitting the experimental data of high strength steels. Liu et al. [31] proposed an expression in the form of Basquin equation for predicting the $S-N$ curves based on the fatigue strengths at $10^{6}$ cycles and at $10^{9}$ cycles. Lai et al. [32] presented a unified model, which provides the prediction of fatigue behavior of hardened steels in different regimes, that is, low cycle fatigue regime quantified by the tensile strength, high cycle fatigue regime obeying Basquin's law and VHCF regime featured by the fisheye and FGA surrounding an initiating inclusion on the fracture surface. A combination of the deterministic model with a stochastic model describing the inclusion size distribution allows prediction of fatigue strength and the associated reliability of a steel component. Sun et al. [33] developed a model for estimating the fatigue life of high-strength steels in high cycle and VHCF regimes with fisheye mode failure based on the cumulative fatigue damage, which takes into account the inclusion size, FGA size and tensile strength of materials. We $[9,10]$ developed a model to investigate the competition between surface and subsurface crack initiation at VHCF regime, and showed that high strength steels with fine grain size tend to initiate crack in the subsurface, whereas surface notch and environmental medium will lead to surface crack initiation. However, models to predict $S-N$ curves in VHCF regime in different environmental media are still lacking due to the complicated crack initiation mechanisms. Recently, new models have been further proposed to predict or estimate the fatigue life for high cycle and VHCF regimes by taking into account the failure mechanism and cumulative damage characteristics [34-37].

In this paper, the process of crack initiation and propagation for a high strength steel in environmental media in VHCF regime is investigated. The specimens of a structural steel were subjected to rotary bending up to VHCF regime in the environments of laboratory air, fresh water and $3.5 \% \mathrm{NaCl}$ aqueous solution, respectively. The influence of environmental media on the variation of fatigue strength and cracking process is presented. Based on the experimental observations, a model is proposed to study the $S-N$ curves of the material in high cycle and VHCF regimes in different media.

\section{Material and experimental method}

In this paper, hour-glass type specimens (Fig. 1a) of a structural steel $40 \mathrm{Cr}$ (main compositions: $0.4 \% \mathrm{C}$ and $1 \% \mathrm{Cr}$ ) were tested with a rotary bending machine operating at a frequency of $52.5 \mathrm{~Hz}$ and the testing environments were of three types: laboratory air, fresh water and $3.5 \% \mathrm{NaCl}$ aqueous solution, respectively, so as to investigate the influence of environmental medium on the variation of fatigue strength and cracking process. The average size of original 


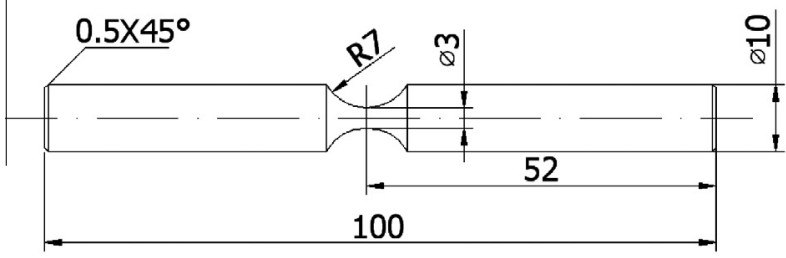

(a)

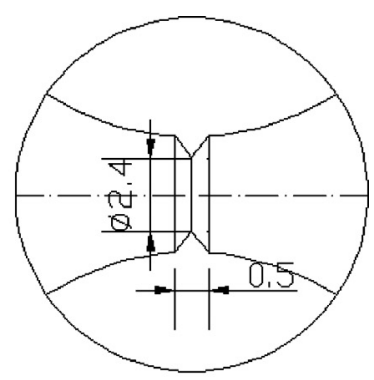

(b)

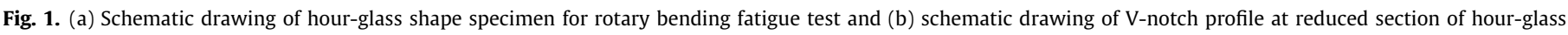
specimen shown in (a), with notch depth of $0.3 \mathrm{~mm}$. (dimensions in $\mathrm{mm}$ ).

austenite grains is $11.2 \mu \mathrm{m}$ and the average yield stress is $1501 \mathrm{MPa}$. Vickers microhardness indentation on the heat-treated specimen gives the average value of $545 \mathrm{kgf} / \mathrm{mm}^{2}$ with the uniform distribution over the specimen cross-section. The $\mathrm{pH}$ values of fresh water and $3.5 \% \mathrm{NaCl}$ aqueous solution are 7.70 and 7.47 , respectively. In addition, the hour-glass type specimens with $\mathrm{V}$ notch at the reduced section, as shown in Fig. 1(b), were also used to investigate the process of fatigue crack propagation. Based on the fatigue test data and scanning electron microscopy (SEM) observations of fracture surfaces, the effect of environment on the fatigue behavior at high cycle and VHCF regimes was examined. The mechanism of crack initiation and propagation under different environmental media was discussed.

\section{3. $S-N$ curves}

For specimens tested in laboratory air (triangular symbols in Fig. 2), single crack originated from the surface of the specimens with fatigue life less than $10^{7}$ loading cycles and the corresponding stress levels are above $700 \mathrm{MPa}$, whereas the crack started from subsurface for the specimens with fatigue life beyond $10^{7}$ loading cycles and the stress levels are below $700 \mathrm{MPa}$. SEM observations showed that for the specimens cyclically fractured in laboratory air, the crack origination is due to a single origin from either surface or subsurface initiation.

For fatigue testing in fresh water, a similar stepwise $S-N$ curve is presented (square symbols in Fig. 2), but the stress of transition

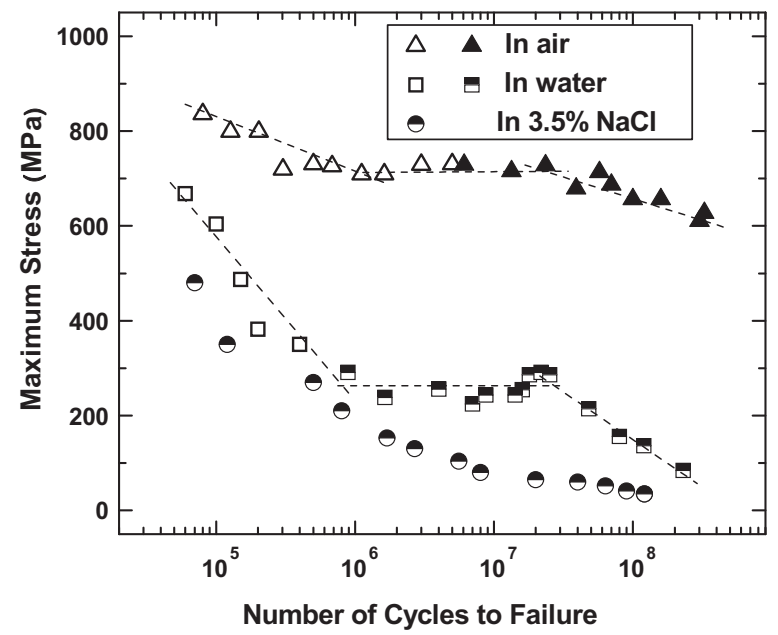

Fig. 2. $S-N$ curves for specimens tested at three environments: laboratory air, fresh water and $3.5 \% \mathrm{NaCl}$ aqueous solution, showing fatigue strength substantially reduced due to the presence of aqueous solutions, and showing relevant crack origination mode, with hollow symbols representing surface mode crack origination, solid symbols representing subsurface mode crack origination and semi-solid symbols representing mixed mode crack origination. part in the $S-N$ curve is dramatically decreased. The maximum stress with respect to the failure cycles of $10^{5}$ is $600 \mathrm{MPa}$, which is about $70 \%$ of the value obtained in the laboratory air testing. When failure cycles extends to $5 \times 10^{5}$, the corresponding maximum stress is $350 \mathrm{MPa}$ for water medium testing, which is only half of the value obtained in the laboratory air testing. In the transition part, the average value of the maximum stress (260 MPa) is only $36 \%$ of that given in laboratory air testing case (720 MPa). In the VHCF regime, the difference of the maximum stress between the two cases is even large. The big difference of fatigue strength presented by these two $S-N$ curves implies that the environmental effect of fresh water on the degradation of fatigue strength is remarkable. In the case of fatigue testing in water medium, for fatigue lives shorter than $5 \times 10^{5}$ loading cycles, the crack origination mode observed by SEM is surface related initiation, the same as the case of laboratory air. For fatigue lives beyond $5 \times 10^{5}$ cycles, namely high cycle fatigue and VHCF regimes, the crack origination observed is a mixed mode of surface related and subsurface initiation.

For the fatigue tests in $3.5 \% \mathrm{NaCl}$ aqueous solution, the $S-N$ curve (lower part of Fig. 2) displays a continuously descending shape. The fatigue strength is even lower than that tested in water medium from low cycle to VHCF regime, implying that the effect of $3.5 \% \mathrm{NaCl}$ aqueous solution on the degradation of fatigue strength for the structural steel is more remarkable than that of water medium. For the case of fatigue testing in $3.5 \% \mathrm{NaCl}$ aqueous solution (circular symbols in Fig. 2), the crack origination observed by SEM for all the specimens is the mixed mode of surface related and subsurface initiation.

The fatigue strength in aqueous solution is substantially lower than that in laboratory air and the reduction increases gradually with decreasing stress level. It is also seen from Fig. 2 that the ratio of the applied maximum stress for the case of tested in fresh water to that in air for the failure life of $10^{7}$ is about $34 \%$. This ratio decreases to $21 \%$ in the vicinity of $10^{8}$ failure cycles. For the case of tested in $3.5 \% \mathrm{NaCl}$ aqueous solution, the fatigue strength decreases even more dramatically. The ratio of the applied maximum stress for the case of tested in $3.5 \% \mathrm{NaCl}$ aqueous solution to that in air is only $10 \%$ for $10^{7}$ failure cycles, and is even lower of $5.8 \%$ in the vicinity of $10^{8}$ failure cycles. The above description is expressed as:

$\left.\frac{\sigma_{\max }^{\mathrm{w}}}{\sigma_{\max }^{\mathrm{a}}}\right|_{10^{7}}=\left.0.34 \quad \frac{\sigma_{\max }^{\mathrm{w}}}{\sigma_{\max }^{\mathrm{a}}}\right|_{10^{8}}=0.21$

and

$\left.\frac{\sigma_{\max }^{\mathrm{s}}}{\sigma_{\max }^{\mathrm{a}}}\right|_{10^{7}}=\left.0.10 \frac{\sigma_{\max }^{\mathrm{s}}}{\sigma_{\max }^{\mathrm{a}}}\right|_{10^{8}}=0.058$,

where $\sigma_{\max }^{\mathrm{w}}$ is the applied maximum stress for the tests in fresh water, $\sigma_{\max }^{\mathrm{s}}$ is the applied maximum stress for the tests in $3.5 \% \mathrm{NaCl}$ aqueous solution, and $\sigma_{\max }^{\mathrm{a}}$ is the applied maximum stress for the tests in laboratory air. 


\section{Fractography for specimens tested in air}

For the specimens tested in laboratory air, all the fatigue fracture surfaces of both surface initiation and subsurface initiation modes present the morphology of three regions as shown in Fig. 3(a). Region A [Fig. 3(b)] is crack initiation and early propagation zone, in which crack propagation velocity is very slow to produce a relatively smooth fracture surface with transgranular cleavage-like morphology and fatigue striations. This region is responsible for a substantially large part of total fatigue life. As shown in Fig. 3(b), crack initiated at the subsurface of specimen at VHCF regime, forming a fisheye pattern originated from a nonmetallic inclusion with main chemical compositions examined as $\mathrm{Al}, \mathrm{Ca}$ and $\mathrm{O}$. The average size of the inclusion located inside the fisheye and as a crack origin is $12 \mu \mathrm{m}$, obtained from 10 measurements. Region B is crack steady and relatively fast growth zone and Fig. 3(c) is a local micrograph of this zone showing quasi-cleavage morphology. Region $\mathrm{C}$ is final fracture zone and the fracture surface presents the ordinary morphology of dimple pattern [Fig. 3(d)].

Regarding the inner boundary as crack tip for Regions A and B, one may calculate the stress intensity factor $K_{\mathrm{I}}$ with the following formula:

$K_{\mathrm{I}}=F \sigma_{\mathrm{a}} \sqrt{\pi a}$

In the calculation, Region $A$ is regarded as elliptical shape and Region B is regarded as circular shape [9]. The values of $K_{\mathrm{I}}$ almost keep constant at $16 \mathrm{MPa}^{1 / 2}$ from high cycle to $\mathrm{VHCF}$ regime for Region A. The values of $K_{\mathrm{I}}$ for Region B are between 35 and
$60 \mathrm{MPa} \mathrm{m}^{1 / 2}$, which correspond to the material fracture toughness. The values of $K_{\mathrm{I}}$ for Region A and B are used to calculate the crack tip plastic zone size based on the expression:

$r_{\mathrm{p}}=\frac{1}{3 \pi}\left(\frac{\Delta K}{\sigma_{\mathrm{s}}}\right)^{2}$

The plastic zone size for Region A is about $12.1 \mu \mathrm{m}$, which approximately equals to one grain size. In the crack initiation and early propagation stage (Region $A$ ), grain boundary serves as microstructural obstacle. In Region $B$, the plastic zone size ranges between $57.7 \mu \mathrm{m}$ and $169.5 \mu \mathrm{m}$. As the increase of the plastic zone size, the crack propagation rate increases significantly. In Region C, as the increase of the crack driving force and decrease of the ligament of the specimen, the specimen displays a plane stress state. Thus, the fracture morphology shows a shear fracture with an angle of $45^{\circ}$ along the tensile direction. For the tested specimens, the fracture is plane strain condition in the crack initiation stage and the crack tip has a high constraint as a result of the small plastic zone size and high stress triaxiality. With the decrease of ligament in Region C, the crack tip has a constraint loss which causes large plastic deformation.

It is noted that the fatigue life consumed by FGA within a fisheye for high strength steels constitutes a significant portion of relevant total fatigue life, which is further confirmed by our recent investigation [6]. For a high strength steel, the size of FGA ranges from 40 to $100 \mu \mathrm{m}$ and the size of fisheye is between 100 and $300 \mu \mathrm{m}$ [6].

Normally, the size of fisheye comprises about 10 grains, and the size of FGA comprises between 2 and 4 grains. It is proposed that
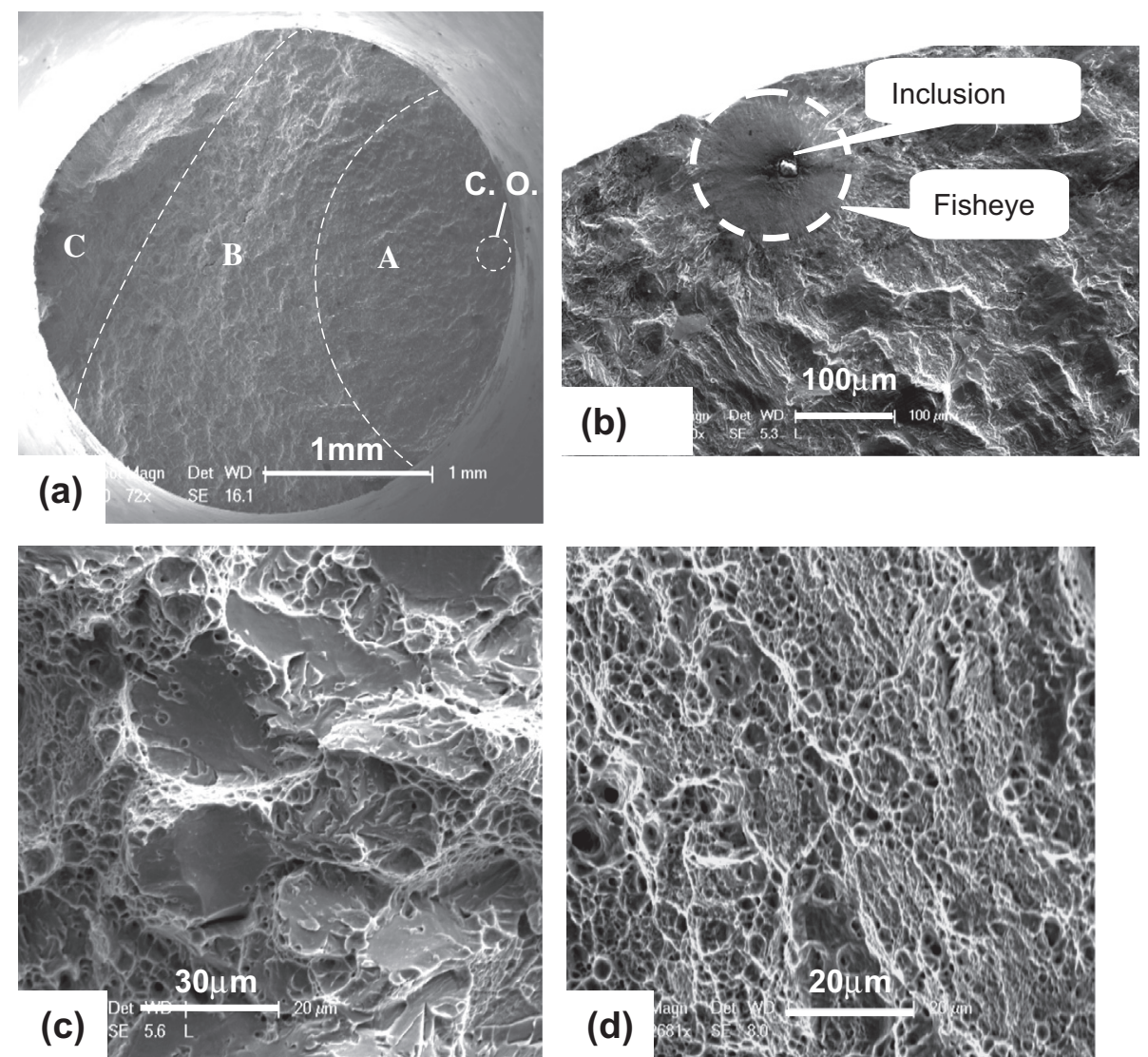

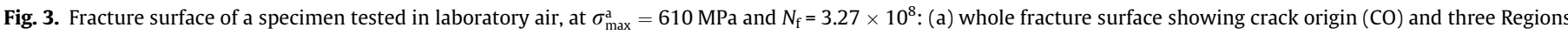

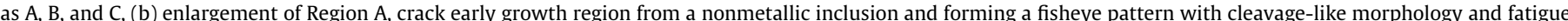

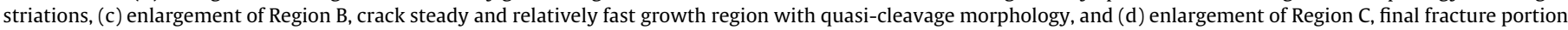
with dimple morphology. 

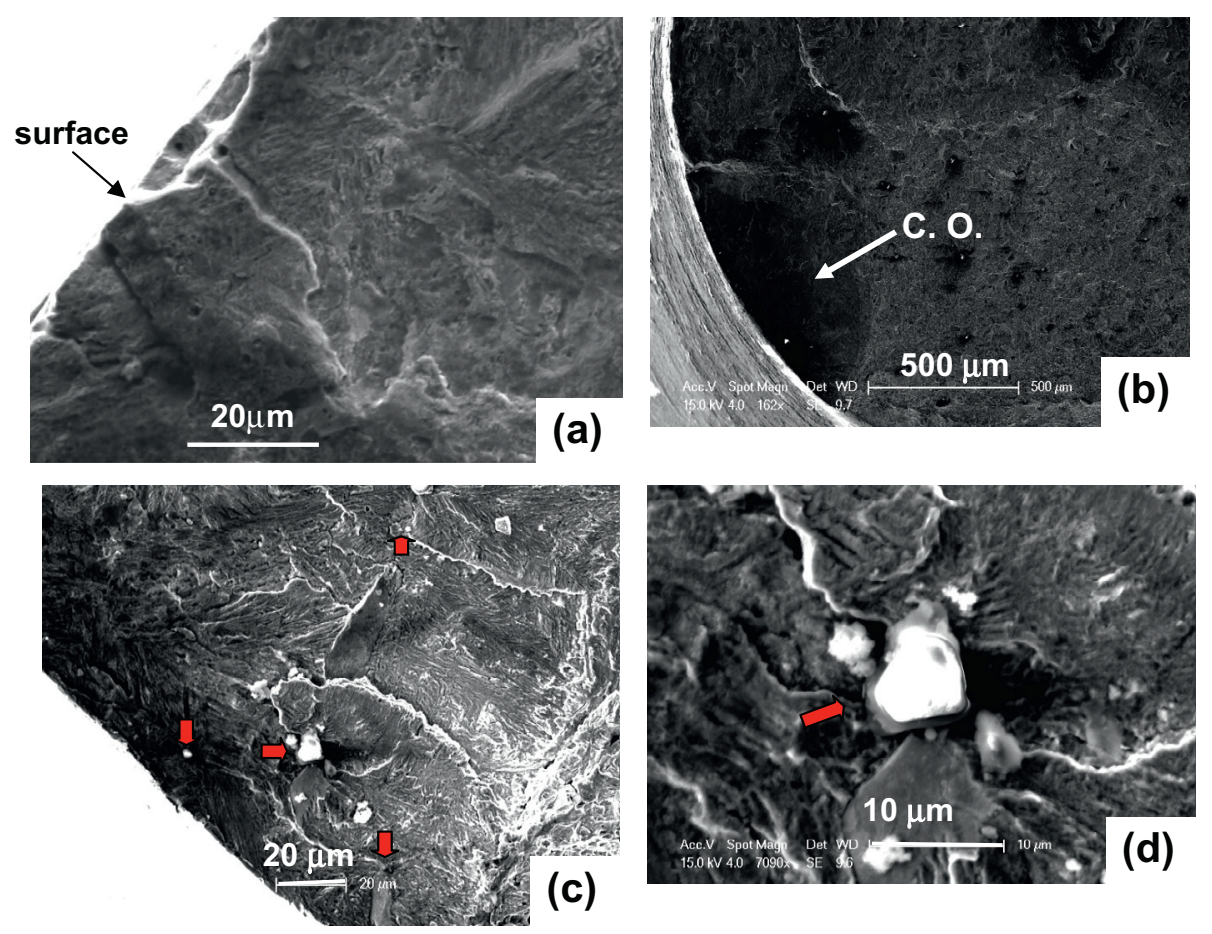

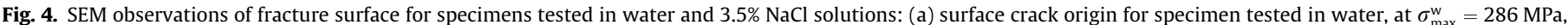

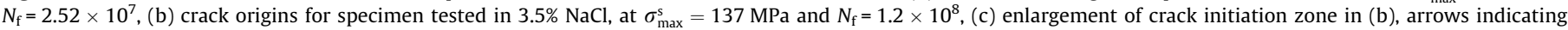
inclusions, and (d) magnification of crack initiation from an inclusion in (c).

the size of FGA is the intrinsically characteristic dimension of crack initiation for VHCF [6], with $\Delta K_{\mathrm{FGA}}=\Delta K_{\mathrm{th}}[6,13]$. The mechanism of crack initiation and early propagation from subsurface for VHCF due to fisheye (containing FGA) defers from that of surface short cracks. The latter is attributed to the PSBs induced by localized plastic deformation. For the case of VHCF with subsurface crack initiation originated from inclusions, the nominal stress is below the value of conventional fatigue limit and the localized surface slip deformation becomes un-activated. Thus, the site of subsurface inclusion becomes the weak point to act as a crack origin after a large number of loading cycles. Therefore, there is a competition process of crack initiation from surface or subsurface $[9,10]$. Indeed, the micro mechanism of crack initiation from subsurface due to nonmetallic inclusions is still not very clear, which needs further in-depth investigations.

It is sure that the size of the inclusion responsible for crack initiation is vital with respect to the cracking mechanism and to the fatigue life [5,33]. In most cases, the inclusion size is smaller than the FGA size. Thus, the morphology of FGA appears and the smaller inclusion leads to a longer fatigue life due to the larger capacity for related cracking within FGA. For some other cases, the size of inclusion responsible for crack initiation is larger than the intrinsic FGA size. Thus, the condition for FGA formation is over, which leads to a relatively smaller value of total fatigue life. Also, other factors of inclusion propensity, interface cohesion between inclusion and matrix, etc. are of influence on the behavior of crack initiation and therefore on the total fatigue life.

\section{Fractography for specimens tested in environmental media}

The observations show that the crack initiation in aqueous solution is of multiple crack origins (Fig. 4b) with surface (Fig. 4a) and subsurface (Fig. 4c) mixed mode. This indicates that the aqueous solution promotes the surface crack initiation. As an example, subsurface crack initiation at nonmetallic inclusions is observed for specimens tested in the aqueous solution in VHCF as shown in Fig. 4(b-d), where the crack initiated at several small nonmetallic inclusions. The diameter of the inclusions is about $3.5 \mu \mathrm{m}$, and the initiated small cracks coalescenced to form an initial crack. This implies that in environmental media, attention should be paid to both small and large inclusions with regard to the increase of fatigue life, although the coalescence effect of the cracks on the fatigue behavior still needs a further study. In addition, it is commonly believed that the maximum inclusion size exists, below which no crack would initiate at inclusions. The maximum inclusion sizes are $5 \mu \mathrm{m}$ [38], $8 \mu \mathrm{m}$ [39] and $15 \mu \mathrm{m}$ [40] for different steels tested in air. Yang et al. [41] proposed an empirical expression to calculate the maximum size for high strength steel, i.e. the critical inclusion size (CIS) by correlating the relation between fatigue strength and Vickers hardness:

$\mathrm{CIS}=\alpha\left(1+\frac{120}{\mathrm{HV}}\right)^{6}$

where $\alpha$ is 0.813 for surface, 0.528 for subsurface and 0.969 for interior inclusions. In this study, CIS for interior inclusions is calculated to be $3.2 \mu \mathrm{m}$ according to Eq. (5). This is in general agreement with the experimental observations of previous ones.

For the case of fatigue tested in water and $3.5 \% \mathrm{NaCl}$ aqueous solution, the crack origination observed by SEM is mainly the surface related initiation. Additionally, unlike the single crack origin for specimens tested in air, multiple fatigue crack origins were observed, and the fracture surface morphology for fatigue crack steady growth zone is predominantly intergranular, as shown in Fig. 5(a). From the measurements on SEM micrographs, the ratio of intergranular morphology is about $75 \%$ for the specimens tested in water, indicating that in water medium, fatigue crack growth along grain boundaries is a major mechanism. For the specimens tested in $3.5 \% \mathrm{NaCl}$ aqueous solution, the intergranular morphology is about $90 \%$ in fatigue crack steady growth zone, indicating that crack growth along grain boundaries is a dominant 

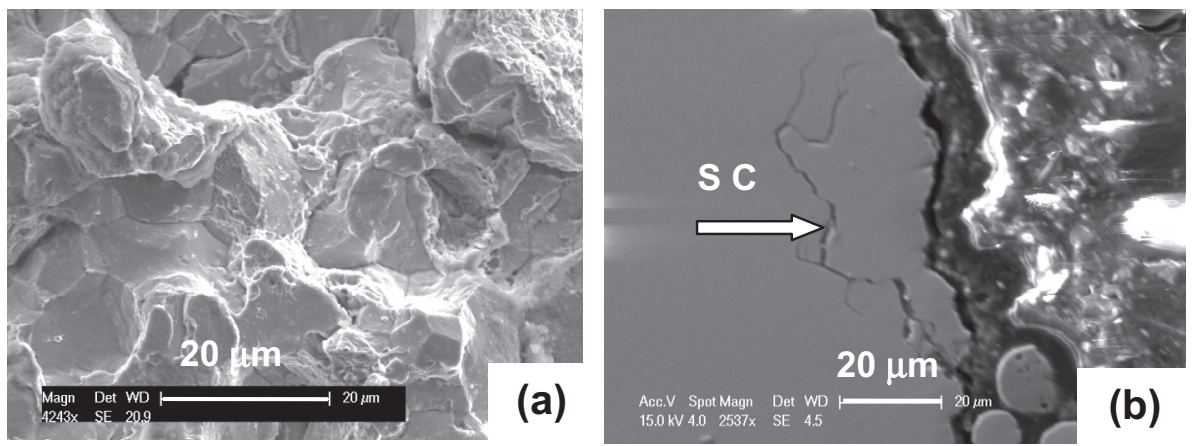

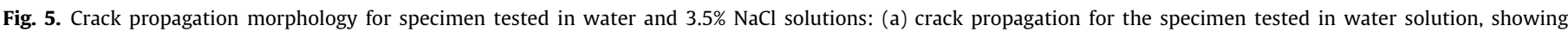

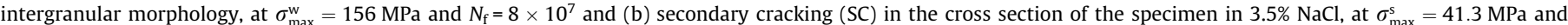
$N_{\mathrm{f}}=9 \times 10^{7}$

mechanism. Fig. 5(a and b) also show secondary cracks along grain boundaries and cross section of the specimen, which is the phenomenon of grain boundary embrittlement due to the aqueous environmental effect. The presence of widespread secondary cracks is observed in the fatigue crack propagation period for the cases tested in water and in $3.5 \% \mathrm{NaCl}$ aqueous solution, which is the damage evidence of environmental media on the material.

\section{Crack propagation process for specimens tested in $3.5 \% \mathrm{NaCl}$ solution}

In addition to the SEM observation on the fracture surface of the broken specimens after fatigue testing, we designed a specific method for further examination of fatigue crack propagation process by taking advantage of low temperature breaking technique, for which a group of 7 specimens were cyclic loaded in $3.5 \% \mathrm{NaCl}$ aqueous solution at the loading value of $\sigma_{\max }^{\mathrm{s}}=22.3 \mathrm{MPa}$. The specimens used for this examination are also hour-glass type but with a V-notch at the reduced section as shown in Fig. 1(b). One of the specimens was loaded to fatigue failure, and the other ones were controlled to terminate at different loading cycles (in sequence) before failure. Then the unloaded specimens were broken by means of low temperature fracture technique, i.e. the unloaded specimen was broken immediately after immersed in the liquid nitrogen for about $20 \mathrm{~min}$, such that the morphology produced during the process of fatigue crack propagation can be separated by the morphology formed at low temperature fracture. The loading cycles of the specimens, which are ready for the observation of fatigue crack propagation, are listed in Table 1. Before the observation on fracture surface of fatigue unloaded specimens, we checked and confirmed that the low temperature fractography of the specimen without fatigue testing is normally cleavage and quasi-cleavage morphology. One important aspect is the observation on the unloaded specimens, for which the failure cycles of specimen $S_{\mathrm{f}}$ is $8 \times 10^{7}$ at $\sigma_{\max }^{\mathrm{s}}=22.3 \mathrm{MPa}$. Fig. 6 is the SEM photograph of whole fracture surface for specimen $S_{\mathrm{f}}$. It is observed that the typical morphology of multiple crack origins prevails at specimen surface or subsurface covering almost the circumference of the notch root, and that a large portion of fracture surface resulted from corrosion fatigue cracking, which is $65.2 \%$ obtained from image anal-

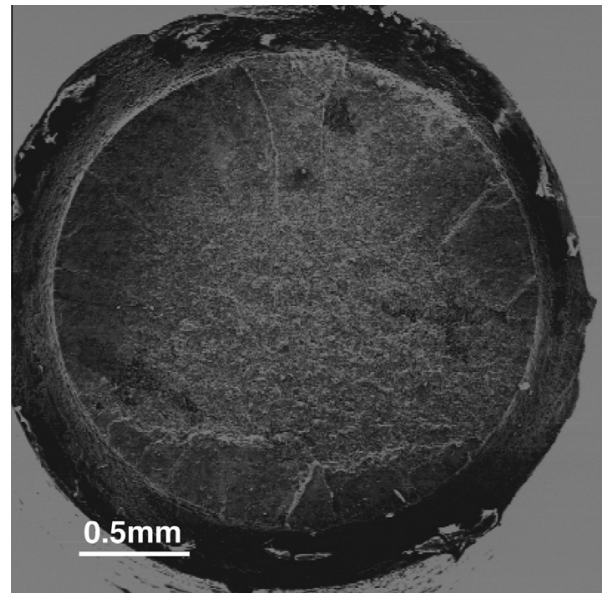

Fig. 6. SEM photograph of whole fracture surface for V-notch specimen $S_{\mathrm{f}}$, showing multiple crack origins and a large portion of cross section resulted from fatigue cracking.

ysis. The observations on unloaded specimens show clear evidence of fatigue crack initiation and early growth at surface and subsurface in the circumference of V-notch specimen. The fraction of fatigue cracking surface, as listed in Table 1, is small for the specimen unloaded after $10^{5}$ cycles of loading and it increases with fatigue loading cycles until failure. Fig. 7( $a$ and $b$ ) are two examples of the specimens unloaded after fatigue cycling of $6 \times 10^{6}$ and $5 \times 10^{7}$, namely specimens $S_{4}$ with cracking area fraction of $24.3 \%$ and $S_{6}$ with that of $48 \%$, respectively. The cracking area fraction of 7 specimens measured by image analysis method increases with the number of loading cycles.

The variation of crack area fraction in the environmental media is attributed to the crack propagation mechanism in the solution. Under aqueous environment, the failure mechanism of high strength steel has been widely confirmed as hydrogen induced embrittlement [42-45]. Hydrogen effect is superimposed by triaxial stress state or stress concentration due to the material heterogeneity to cause stress corrosion cracking or corrosion fatigue

Table 1

Loading cycles and cracking area fraction of $\mathrm{V}$-notch specimens tested in $3.5 \% \mathrm{NaCl}$ aqueous solution.

\begin{tabular}{|c|c|c|c|c|c|c|c|}
\hline Specimen $^{\mathrm{a}}$ & $S_{1}$ & $S_{2}$ & $S_{3}$ & $S_{4}$ & $S_{5}$ & $S_{6}$ & $S_{\mathrm{f}}$ \\
\hline Cycles & $1 \times 10^{5}$ & $5 \times 10^{5}$ & $2 \times 10^{6}$ & $6 \times 10^{6}$ & $1 \times 10^{7}$ & $5 \times 10^{7}$ & $8 \times 10^{7}$ \\
\hline Cracking area fraction (\%) & 3.25 & 7.84 & 13.3 & 24.3 & 41.1 & 48 & 65.2 \\
\hline
\end{tabular}

a Specimen $S_{\mathrm{f}}$ broke after cyclic loading, other specimens stopped loading at the given loading cycle then broken in liquid nitrogen. 

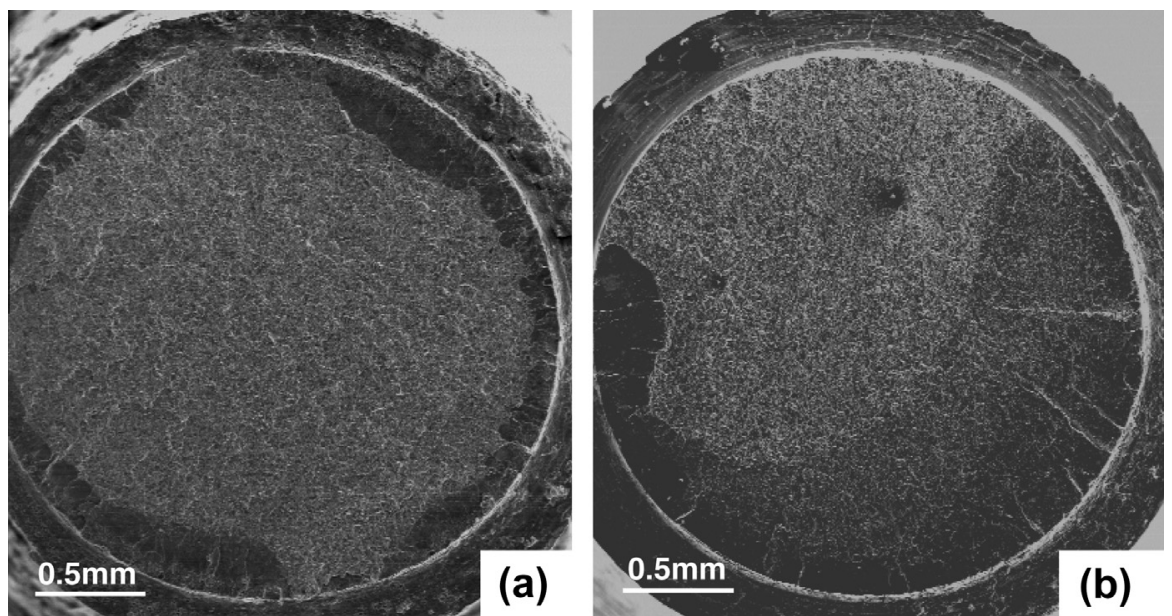

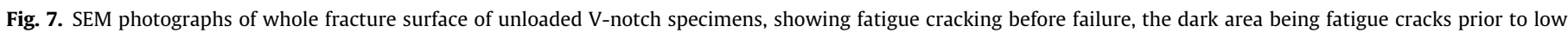
temperature fracture: (a) specimen $S_{4}$ and (b) specimen $S_{6}$.

$[44,45]$. In the process of hydrogen induced failure, the diffusion and concentration of hydrogen is critical to the fatigue damage. It is obvious that a longer time period exposed to the aqueous environment while cyclic loading will lead to deeper hydrogen diffusion into the specimen and therefore more severe effect of the test environment superimposed on the mechanical loading, which is the qualitative explanation of the cracking area increase with loading cycles and the difference of fatigue strength between the cases tested in air and in aqueous solution is large at higher failure lives, i.e. the maximum stress is inversely proportional to fatigue failure cycles when introducing the aqueous environment as previously shown in Eqs. (1) and (2), and Fig. 2.

\section{Model for $S-N$ curve prediction in different environmental media}

It is known that the crack initiation related to inclusions is attributed to the weak cohesive state between inclusion and matrix. Under cyclic loading, a crack may easily form due to the interface debonding and grow into the matrix. In such a case, the subsurface crack initiation cycle $N_{\mathrm{i}}$ is [46]:

$N_{\mathrm{i}}=\frac{4 l w_{i}}{\Delta U_{\mathrm{i}}}$,

where $w_{\mathrm{i}}$ is surface energy related to subsurface crack initiation, $l$ is grain radius and $\Delta U_{\mathrm{i}}$ is unit increment of energy for subsurface crack initiation. $w_{\mathrm{i}}$ and $\Delta U_{\mathrm{i}}$ are functions of grain radius $l$, inclusion radius $r(\psi=r / l)$, stress amplitude $\Delta \sigma$ and the resistance of dislocation movement $k(\varphi=0.5 \Delta \sigma / k) . \Delta U_{\mathrm{i}}$ is written as:

$\Delta U_{\mathrm{i}}=\frac{\Delta \widetilde{U}(2 k)^{2} r^{2}}{A}$,

where

$A=\frac{\mu}{2 \pi(1-v)}$,

and

$\Delta \widetilde{U}=\frac{\varphi \Delta \tilde{\gamma}_{1}^{\mathrm{T}}-\Delta \tilde{\gamma}_{1}^{\mathrm{L}}+\varphi^{2}}{2}$,

with

$\Delta \tilde{\gamma}_{1}^{\mathrm{T}}=\beta \varphi\left(2 \tilde{c}^{2}-1\right)+\frac{1}{\pi}\left(3 \sqrt{\tilde{c}^{2}-1}-2 \widetilde{I}\right)-\frac{1}{4}$,

$$
\begin{aligned}
& \Delta \tilde{\gamma}_{1}^{L}=\frac{2 \beta \varphi}{\pi}\left[\tilde{c}^{2} \cos ^{-1}\left(\frac{1}{\tilde{c}}\right)-\sqrt{\tilde{c}^{2}-1}+\frac{\pi}{2}\left(\tilde{c}^{2}-1\right)\right] \\
& +\frac{2}{\pi^{2}}\left\{\sqrt{\tilde{c}^{2}-1}\left[\pi+\cos ^{-1}\left(\frac{1}{\tilde{c}}\right)\right]+2 \ln \left(\frac{1}{\tilde{c}}\right)-\pi \widetilde{I}\right\} \text {, } \\
& \beta=1-\frac{1}{2 \varphi}-\frac{1}{\pi \varphi} \cos ^{-1}\left(\frac{1}{\tilde{c}}\right) \\
& \tilde{c}=\frac{\psi}{2(\psi+2)}\left[1+\left(1+\frac{2}{\psi}\right)^{2}\right] \text {, }
\end{aligned}
$$

and

$\widetilde{I}=\frac{1}{\pi} \int_{1}^{\tilde{c}} \frac{t^{2}}{\sqrt{t^{2}-1}} \ln \left|\frac{t \sqrt{\tilde{c}^{2}-1}+\sqrt{\tilde{c}^{2}-t^{2}}}{t \sqrt{\tilde{c}^{2}-1}-\sqrt{\tilde{c}^{2}-t^{2}}}\right| d t$.

Thus,

$N_{\mathrm{i}}=\frac{4 l W_{i}}{\Delta U_{i}}=\frac{4 l A W_{i}}{\Delta \widetilde{U}(2 k)^{2} a^{2}}=\frac{4 A W_{i}}{\psi^{2} \Delta \widetilde{U}(2 k)^{2} l}$,

For the normalization of $N_{\mathrm{i}}, \widetilde{N}$ is defined as

$\widetilde{N}=\frac{A W_{i}}{(2 k)^{2} l}$

Thus,

$n_{i}=\frac{N_{i}}{\widetilde{N}}=\frac{4}{\psi^{2} \Delta \widetilde{U}}$

The variation of $n_{\mathrm{i}}$ with $\varphi$ and $\psi$ are demonstrated in Fig. 8 by assuming $\varphi$ to be $1.1,1.2,1.4,2$ and 4 , and $\psi$ varying from 0 to 2 . It is shown that fatigue life $n_{\mathrm{i}}$ increases with the decrease of $\varphi$, i.e. the decrease of fatigue loading $\Delta \sigma$ or the increase of the resistance of dislocation movement $k$. For a given loading state ( $\varphi$ in constant), fatigue life generally decreases with the increase of $\psi$, namely the increase of inclusion size $r$ or the decrease of grain size $l$. The trends are in agreement with the experimental observations. Yang et al. $[41,47]$ observed that the fatigue life decreases with the increase of inclusion size for an alloy steel. It is widely observed that the fatigue life increases with the decrease of applied loading. Zhao et al. [12] found that the fatigue life increases with the resistance of dislocation movement, i.e. the yield stress of material.

For fatigue crack initiation at surface, by considering the surface crack factor and half cycling process [46], surface crack initiation cycle $N_{\mathrm{s}}$ is 


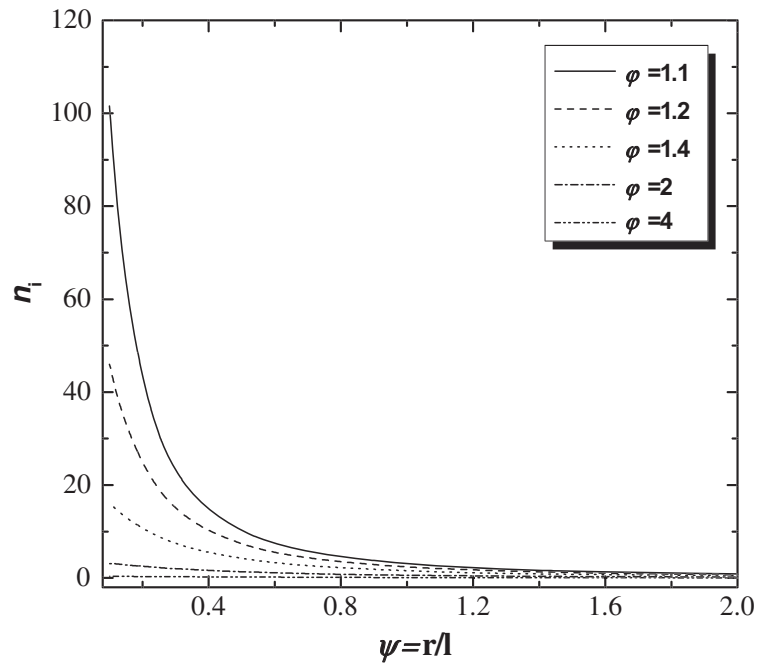

Fig. 8. Predicted $S-N$ curves of subsurface crack initiation for specimens tested in air, for different loading levels and material properties.

$N_{s}=\frac{2 l w_{s}}{\Delta U_{s}}$

where $\Delta U_{s}$ is the unit increment of energy for surface crack initiation, $w_{\mathrm{s}}$ is surface energy related to surface crack initiation. $w_{\mathrm{s}}$ and $\Delta U_{s}$ are functions of the grain radius $l$, the inclusion radius $r$, the stress amplitude $\Delta \sigma$ and the resistance of dislocation movement $k$. And $\Delta U_{\mathrm{s}}$ is approximated $[10,46]$ as:

$\Delta U_{\mathrm{s}} \approx \frac{1.25}{8 A}(\Delta \sigma-2 k)^{2}(2 l)^{2}$.

Thus,

$N_{\mathrm{s}}=\frac{4 A w_{\mathrm{s}}}{1.25 l(\Delta \sigma-2 k)^{2}}$.

$N_{\mathrm{s}}$ is also normalized by $\widetilde{N}$ :

$n_{\mathrm{s}}=\frac{N_{\mathrm{s}}}{\widetilde{N}}=\frac{4}{1.25(\varphi-1)^{2} k_{\mathrm{w}}}$.

Note that both $n_{\mathrm{s}}$ and $n_{\mathrm{i}}$ are the functions of $\varphi$ and $\psi$. In short, Eqs. (17) and (21) are derived for the calculation of the fatigue life for crack initiation at surface or at subsurface in different environmental media.
For the case tested in air, $k_{\mathrm{w}}$ is taken as 3 in the calculation [48]. For the case tested in $3.5 \% \mathrm{NaCl}$ solution, $k_{\mathrm{w}}$ is taken as 25 times of that in air, i.e. 75 , from the relationship of $K_{\mathrm{IC}}$ in air and the aqueous solution [10,49]. The fatigue life for surface crack initiation $n_{\mathrm{s}}$ and subsurface crack initiation $n_{\mathrm{i}}$ in air as a function of $\varphi$ and $\psi$ is compared in Fig. 9(a). It is seen that the subsurface crack initiation life is higher than the surface crack initiation life for the same high value of $\varphi$ (high loading or low material yield stress). Thus, surface crack initiation occurs much easier in this stage. With decreasing $\varphi$, the surface crack initiation life is higher than the subsurface crack initiation life at the same $\varphi$, which as a consequence leads to the subsurface crack initiation. At points $A, B$ and $C$, the subsurface crack initiation life equals to the surface crack initiation. The three points correspond to the transition plateau in an $S-N$ curve from the subsurface to the surface crack initiation.

Fig. 9(b) compares the surface crack initiation life with subsurface crack initiation life in $3.5 \% \mathrm{NaCl}$ solution. A similar trend as that in air presents. However, the fatigue life for surface crack initiation $n_{\mathrm{s}}$ in $3.5 \% \mathrm{NaCl}$ solution is significantly decreased, which interprets the observation in Section 5 that surface crack initiation even occurs in VHCF regime. This is due to the fact that aqueous media promote surface crack initiation in the competition of surface versus subsurface crack initiation.

It is also seen from Fig. 9( $a$ and $b$ ) that when subjected to the same loading, the fatigue life in air is much longer than that in aqueous media. This explains the characteristics of the $S-N$ curve for the fatigue behavior in Fig. 2. In addition, it is seen that in the transition, there displays no pronounced stepwise tendency. If the uncertainty of the fatigue life is considered, the $S-N$ curves for subsurface and surface fatigue fall into the same scatter band. This is in agreement with the experiment results by Wang et al. [50] obtained from push-pull fatigue test for tool steels, as shown in Fig. 10. Also, the $S-N$ curve in Lai et al. [32] agrees well with our model. The stepwise $S-N$ curve (Fig. 2 for air) obtained from the rotary bending test is partly explained by the smaller "control volume" of the specimen due to the stress gradient along the specimen section. The effect of control volume has a significant impact on the crack initiation and fatigue life of the specimen according to the weakest link concept. It is shown in Fig. 9(a and b) that the loading curves at different $\varphi$ tend to be a plateau with the increasing of fatigue life. This is the threshold for plastic deformation, below which the dislocation is assumed to be locked in this model.
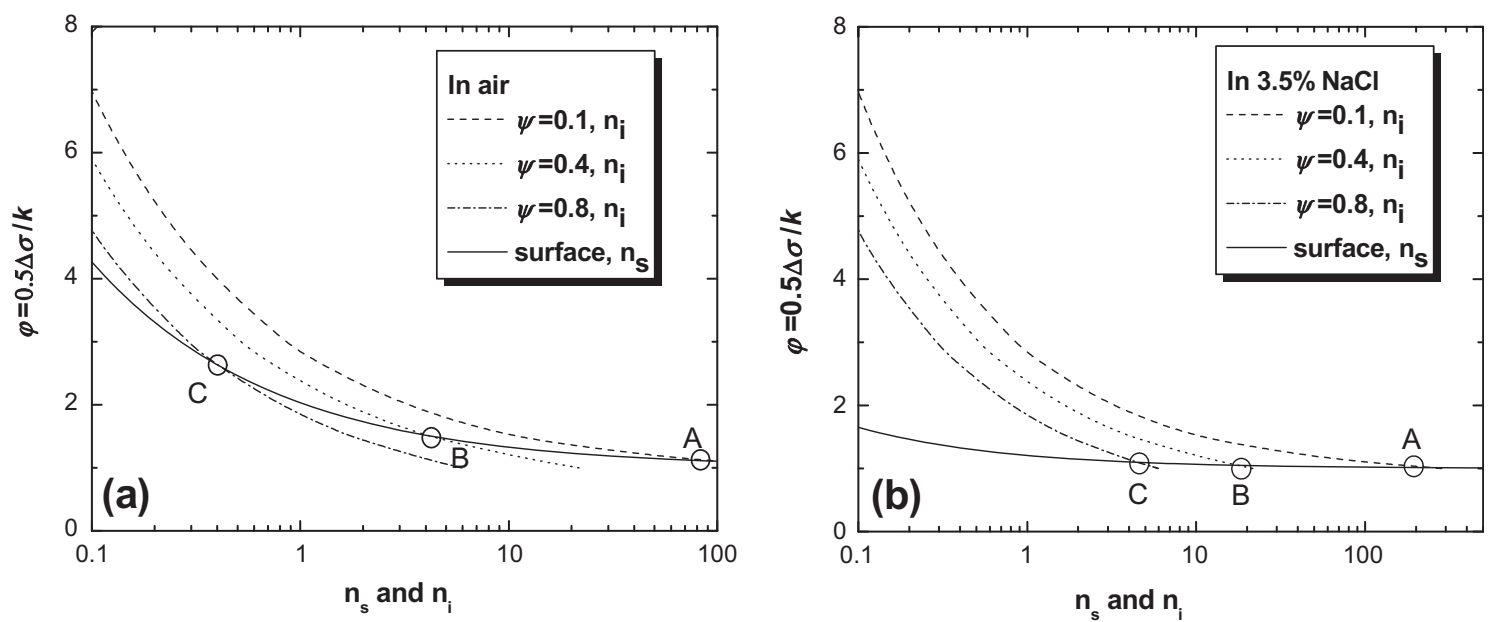

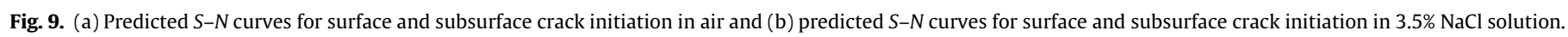




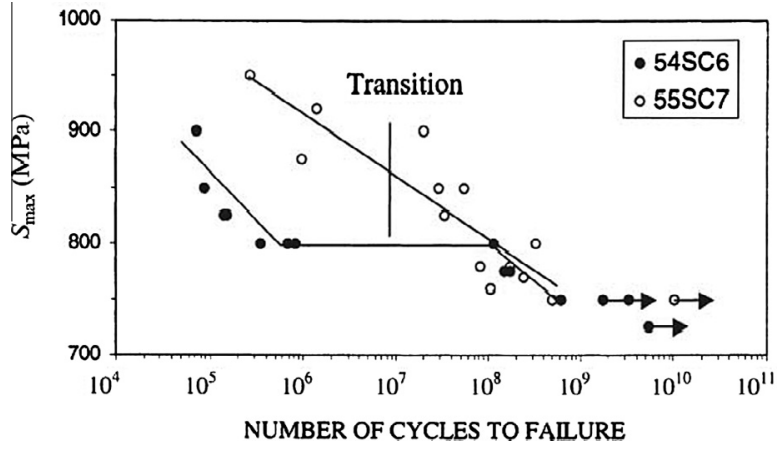

Fig. 10. $S-N$ curves of two tool steels in high cycle and VHCF regimes [50].

In all, this model predicts that fatigue life decreases with the applied loading and inclusion size, whereas it increases with the material yield stress. The plateau of $S-N$ curve corresponding to the transition from surface crack initiation to subsurface crack initiation is predicted. In $3.5 \% \mathrm{NaCl}$ solution, the fatigue life decreases significantly and surface crack initiation occurred even in VHCF regime. The competition of crack initiation at surface and subsurface in different environmental media is also predicted with the model. Note that this model qualitatively predicts $S-N$ curves for surface and subsurface crack initiation in different environmental media. A quantification prediction of $S-N$ curves based on this method is planned.

\section{Conclusions}

Based on this study, the following conclusions are drawn:

(1) During the crack propagation process for specimens tested in air, fracture surface displays three regions with different propagation mechanisms. The formation of different morphologies in these regions is attributed to different crack driving forces and plastic zone sizes (crack tip constraint) ahead of the crack tip.

(2) The values of fatigue strength for specimens tested in water and in $3.5 \% \mathrm{NaCl}$ aqueous solution are significantly decreased compared to that tested in air. The fractography characteristics for specimens tested in aqueous solution are multiple crack originations and intergranular cracking mode with widespread secondary cracks in fatigue crack steady propagation period.

(3) For fatigue testing in water and $3.5 \% \mathrm{NaCl}$ aqueous solution, subsurface crack initiation is observed at small nonmetallic inclusions. They initiated and coalescenced to larger crack.

(4) For fatigue testing in $3.5 \% \mathrm{NaCl}$ aqueous solution, the cracking area fraction of specimens increases with loading cycles, which is attributed to the effect of mechanical cycling superimposed by the corrosive action of environment.

(5) A model is proposed to study the relationship between fatigue life, applied stress and material property in VHCF in different environmental media. This model predicts that fatigue life decreases with the increase of loading and inclusion size, whereas it increases with the material yield stress. In $3.5 \% \mathrm{NaCl}$ solution, the fatigue life decreases significantly and surface crack initiation occurred even in VHCF regime. The competition of crack initiation at surface and subsurface in different environmental media is also predicted with the model. The model prediction is in good agreement with experimental observations.

\section{Acknowledgements}

This work was funded by the National Basic Research Program of China (2012CB937500) and the National Natural Science Foundation of China (Nos. 11172304, 11021262 and 11202210).

\section{References}

[1] Stanzl S, Tschegg E, Mayer H. Lifetime measurements for random loading in the very high cycle fatigue range. Int J Fatigue 1986;8:195-200.

[2] Lukáš P, Kunz L. Specific features of high-cycle and ultra-high-cycle fatigue. Fatigue Fract Eng Mater Struct 2002;25:747-53.

[3] Murakami Y, Yokoyama N, Nagata J. Mechanism of fatigue failure in ultralong life regime. Fatigue Fract Eng Mater Struct 2002;25:735-46.

[4] Bathias C, Paris PC. Gigacycle Fatigue in Mechanical Practice. New York: Marcel Dekker; 2005.

[5] Lei Z, Hong Y, Xie J, Sun C, Zhao A. Effects of inclusion size and location on veryhigh-cycle fatigue behavior for high strength steels. Mater Sci Eng A 2012;558:234-41.

[6] Hong Y, Lei Z, Sun C, Zhao A. Propensities of crack interior initiation and early growth for very-high-cycle fatigue of high strength steels. Int J Fatigue 2014;58:144-51.

[7] Zhou C, Qian G, Hong Y. Fractography and crack initiation of very-high-cycle fatigue for a high carbon low alloy steel. Key Eng Mater 2006;324325:1113-6.

[8] Qian G, Hong Y, Zhou C. Investigation of high cycle and very-high-cycle fatigue behaviors for a structural steel with smooth and notched specimens. Eng Fail Anal 2010;17:1517-25

[9] Hong Y, Zhao A, Qian G, Zhou C. Fatigue strength and crack initiation mechanism of very-high-cycle fatigue for low alloy steels. Metall Mater Trans A 2012;43:2753-62.

[10] Qian G, Zhou C, Hong Y. Experimental and theoretical investigation of environmental media on very-high-cycle fatigue behavior for a structural steel. Acta Mater 2011:59:1321-7.

[11] Qian G, Hong Y. Effects of environmental media on high cycle and very-highcycle fatigue behaviors of structural steel 40Cr. Acta Metall Sin 2009;45:1359-63.

[12] Zhao A, Xie J, Sun C, Lei Z, Hong Y. Effects of strength level and loading frequency on very-high-cycle fatigue behavior for a bearing steel. Int J Fatigue 2012;38:46-56.

[13] Zhao A, Xie J, Sun C, Lei Z, Hong Y. Prediction of threshold value for FGA formation. Mater Sci Eng A 2011;528:6872-7.

[14] Furuya Y, Matsuoka S, Abe T, Yamaguchi K. Gigacycle fatigue properties for high-strength low-alloy steel at $100 \mathrm{~Hz}, 600 \mathrm{~Hz}$, and $20 \mathrm{kHz}$. Scr Mater 2002;46:157-62.

[15] Nishijima S, Kanazawa K. Stepwise $S-N$ curve and fish-eye failure in gigacycle fatigue. Fatigue Fract Eng Mater Struct 1999;22:601-7.

[16] Shiozawa K, Lu L. Very high-cycle fatigue behaviour of shot-peened highcarbon-chromium bearing steel. Fatigue Fract Eng Mater Struct $2002 ; 25: 813-22$

[17] Naito T, Ueda H, Kikuchi M. Observation of fatigue fracture surface of carburized steel. Jpn Soc Mater Sci 1983:32:1162-6.

[18] Naito T, Ueda H, Kikuchi M. Fatigue behavior of carburized steel with internal oxides and nonmartensitic microstructure near the surface. Met Trans A 1984:15A:1431-6.

[19] Atrens A, Hoffelner W, Duerig TW, Allison JE. Subsurface crack initiation in high cycle fatigue in Ti6Al4V and in a typical martensitic stainless steel. Scr Metall 1983;17:601-6.

[20] Stanzl-Tschegg S, Mayer H. Fatigue and fatigue crack growth of aluminum alloys at very high numbers of cycles. Fatigue damage in structural materials III. UK; 17-22 September 2001.

[21] Engler-Pinto CC, Frisch RJ, Lasecki JV, Mayer H, Allison JE. Effect of frequency and environment on high cycle fatigue of cast aluminum alloys. In: Proc VHCF 4. Ann, Arbor; 2007. p. 421-7.

[22] Itoga H, Tokaji K, Nakajima M, Ko H-N. Effect of surface roughness on stepwise $S-N$ characteristics in high strength steel. Int J Fatigue 2003;25:379-85.

[23] Dominguez Almaraz G. Prediction of very high cycle fatigue failure for high strength steels, based on the inclusion geometrical properties. Mech Mater 2008:40:636-40.

[24] Makino T. The effect of inclusion geometry according to forging ratio and metal flow direction on very high-cycle fatigue properties of steel bars. Int J Fatigue 2008;30:1409-18.

[25] Krupp U, Helge K, Christ H, Köster P, Fritzen C. The significance of microstructural barriers during fatigue of a duplex steel in the high- and very-high-cycle-fatigue (HCF/VHCF) regime. Int J Fatigue 2010;32:914-20.

[26] Tokaji K, Ko H-N, Nakajima M, Itoga H. Effects of humidity on crack initiation mechanism and associated $S-N$ characteristics in very high strength steels. Mater Sci Eng A 2003;345:197-206.

[27] Paris P, Marines-Garcia I, Hertzberg R, Donald J. The Relationship of effective stress intensity, elastic modulus and Burgers-vector on fatigue crack growth as associated with "fish eye" gigacycle fatigue phenomena. In: Proc VHCF III Kyoto. Japan; September 2004. p. 1-13. 
[28] Marines-Garcia I, Paris P, Bathias C, Lados D. Fatigue crack growth from small to large cracks on very high cycle fatigue with fish-eye failures. Eng Fract Mech 2008;75:1657-65.

[29] Marines-Garcia I, Paris Paul C, Tada H, Bathias C. Fatigue crack growth from small to long cracks in very-high-cycle fatigue with surface and internal "fisheye" failures for ferrite-perlitic low carbon steel SAE 8620. Mater Sci Eng A 2007;468-470:120-8.

[30] Chapetti M, Tagawa T, Miyata T. Ultra-long cycle fatigue of high-strength carbon steels Part II: Estimation of fatigue limit for failure from internal inclusions. Mater Sci Eng A 2003;356:236-44.

[31] Liu Y, Li Y, Li S, Yang Z, Chen S, Hui W, et al. Prediction of the $S-N$ curves of high-strength steels in the very high cycle fatigue regime. Int J Fatigue 2010;32:1351-7.

[32] Lai J, Lund T, Ryden K, Gabelli A, Strandell I. The fatigue limit of bearing steels Part I: A pragmatic approach to predict very high cycle fatigue strength. Int J Fatigue 2012;37:155-68.

[33] Sun C, Xie J, Zhao A, Lei Z, Hong Y. A cumulative damage model for fatigue life estimation of high-strength steels in high-cycle and very-high-cycle fatigue regimes. Fatigue Fract Eng Mater Struct 2012;35:638-47.

[34] Paolino D, Chiandussi G, Rossetto M. A unified statistical model for $S-N$ fatigue curves: probabilistic definition. Fatigue Fract Eng Mater Struct 2013;36:187-201.

[35] Huang Z, Wang Q, Wagner D, Bathias C, Chaboche J. A rapid scatter prediction method for very high cycle fatigue. Fatigue Fract Eng Mater Struct 2013;36:462-8.

[36] Sun C, Lei Z, Xie J, Hong Y. Effects of inclusion size and stress ratio on fatigue strength for high-strength steels with fish-eye mode failure. Int J Fatigue 2013;48:19-27.

[37] Stepanskiy L. Cumulative model of very high cycle fatigue. Fatigue Fract Eng Mater Struct 2012;35:513-22.
[38] Kiedssling R. Non-metallic inclusions in steel. London: Meteorological Society; 1978. p. 155-65.

[39] Kawada Y, Nakazawa H, Kodama S. Mem fac. Techn Tokyo Metropol Univ, vol 15; 1965. p. 1163-76.

[40] Furuya Y, Hirukawa H, Kimura T, Hayaishi M. Gigacycle fatigue properties of high-strength steels according to inclusion and ODA sizes. Metall Mater Trans A 2007;38A:1722-30.

41] Yang Z, Zhang J, Li S, Li G, Wang Q, Hui W, et al. On the critical inclusion size of high strength steels under ultra-high cycle fatigue. Mater Sci Eng A 2006;427:167-74.

[42] McMahon Jr CJ. Hydrogen-induced intergranular fracture of steels. Eng Fract Mech 2001;68:773-88.

[43] Taha A, Sofronis P. A micromechanics approach to the study of hydrogen transport and embrittlement. Eng Fract Mech 2001;68:803-37.

[44] Nagumo M, Shimura H, Chaya T, Hayashi H, Ochiai I. Fatigue damage and its interaction with hydrogen in martensitic steels. Mater Sci Eng A 2003;348:192-200.

[45] Nagao A, Kuramoto S, Ichitani K, Kanno M. Visualization of hydrogen transpor in high strength steels affected by stress fields and hydrogen trapping. Sc Mater 2001;45:1227-32.

[46] Tanaka T, Mura T. A dislocation model for fatigue crack initiation. J App Mech Trans ASME 1981;48:97-103.

[47] Yang Z, Li S, Zhang J, Zhang J, Li G, Li Z, et al. The fatigue behaviors of zeroinclusion and commercial 42CrMo steels in the super-long fatigue life regime. Acta Mater 2004;52:5235-41.

[48] Venkataraman G, Chung Y, Nakasone Y, Mura T. Free energy formulation of fatigue crack initiation along persistent slip bands: calculation of $S-N$ curves and crack depths. Acta Metall 1990;38:31-40.

[49] Suresh S. Fatigue of materials. Cambridge: Cambridge University Press; 1998.

[50] Wang Q, Berard J, Dubarre A, Baudry G, Rathery S, Bathias C. Fatigue Fract Eng Mater Struct 1999;22:667-72. 